Rev. Elev. Méd. vét. Pays trop., 1972, 25 (2) : 141-153

\title{
Essais de vaccination antibovipestique par voie pernasale de veaux possédant ou non une immunité colostrale
}

\author{
par A. PROVOST (*) et C. BORREDON $\left({ }^{*}\right)$ \\ (avec la collaboration technique de Mme G. DUFAU et de M. Z. N'GALDAM)
}

\section{RESUME}

Se basant sur le fait établi que les anticorps colostraux ne se retrouvent pas dans les muco-anticorps nasaux des veaux encore sous le couvert de l'immunité maternelke transmise, les auteurs ont vacciné de tels animaux par voie nasale avec le virus-vaccin antiseptique de cultures cellulaires ou le virus morbilleux souche MB $113 \mathrm{Y}$, soit par dépôt du matériel vaccinal avec une sonde plastique soit par brum1sation avec un vaporisateur. Le procédé permet limmunisation active de 96 p. 100 des veaux possédant encore un titre sérique résiduel de $\mathrm{TN}_{\overline{5}} \leqslant 0,9$ et de certains possédant plus d'anticorps. La méthode vaut dêtre vulgarisée pour couper le cycle épizootiologique de la peste bovine en réduisant le nombre de veaux réceptifs à la maladie tout en produisant une immunité locale interdisant la réplication du virus.

\section{INTRODUCTION}

Dans une précédente communication (18), on a indiqué que l'activité neutralisante du sérum des bovins vaccinés contre la peste bovine ne se retrouve que rarement dans leur mucus nasal, fait qui permet d'expliquer le portage local du virus bovipestique chez de tels animaux placés au contact de leurs congénères atteints; ces animaux, infectés occultes, peuvent dès lors servir de relais passif ou actif dans la transmission du contage bovipestique. Cette absence de muco-anticorps spécifiques antibovipestiques se retrouve chez les veaux de lait possédant par ailleurs une solide immunité antipestique colostrale: sans être euxmêmes apparemment malades, ils peuvent se contaminer au contact de bovins pestiques et infecter d'autres veaux à immunité antipestique évanescente (19).

(*) I.E.M.V.T., Laboratoire de Recherches vétérinaires de Farcha, B.P. n' 433 , Fort-Lamy, Tchad.
L'attention était ainsi attirée sur le rôle que peuvent jouer les voies aériennes supérieures dans la transmission du contage bovipestique par leur défaut d'immunité locale chez les bovins pourvus d'une immunité humorale. Il devenait logique de tenter d'y faire apparaître des anticorps locaux pour couper le cycle de transmission du virus. De plus, le système sécrétoire immunitaire $\left({ }^{* *}\right)$ des bovins possédant deux aspects, général et local (12), on pouvait se demander s'il n'était pas possible d'imprimer une immunité active nasale, et peut-être générale, aux veaux possédant une immunité antiseptique colostrale, ce qui permettrait de rompre la barrière immunologique que représente le reliquat d'anticorps maternels qu'ils peuvent héberger.

Des essais de vaccination contre la peste

(***) Traduction, au demeurant non euphonique, de l'expression anglaise: Secretory immune system. 
bovine par voie nasale font l'objet de cette communication.

\section{MATERIEL ET METHODES}

\section{Animaux d'expérience}

Deux cents veaux de race zébu arabe, d’âge variable de quelques jours à un an, entrent en expérience. Tous ont absorbé le colostrum de leur mère, pratique heureusement constante en Afrique centrale. Quelques-uns, les plus vieux. portent au moment de l'intervention la trace auriculaire d'une primo-vaccination antérieurement effectuée à un âge non indiqué.

Une prise de sang est pratiquée avant la vaccination puis un mois plus tard. Chez certains, on a collecté le mucus nasal par écouvillonnage.

Les essais se sont déroulés dans leurs villages d'origine, les veaux étant confiés à leurs propriétaires. Quelques-uns ont pu être achetés pour être éprouvés au laboratoire.

Par ailleurs, 7 zébus bororos adultes, importés de R.C.A. (territoire non infecté de peste bovine et où, de ce fait, aucune vaccination n'est pratiquée) servent dans les étables d'isolement du laboratoire de témoins d'un pouvoir pathogène éventuel du virus vaccinal utilisé. Une prise de sang est effectuée avant, puis 28 jours après l'essai.

\section{Vaccins}

Le vaccin antipestique utilisé est la souche atténuée, adaptée aux cultures cellulaires, de PLOWRIGHT et FERRIS (14), aux alentours de son $40^{\circ}$ passage. Le virus a été cultivé, soit en cellules de reins de foetus bovin de $2^{\mathrm{c}}$ passage, soit en cellules de la lignée cellulaire de MADIN et DARBY (lignée MDBK). Le vaccin a été produit selon des normes déjà décrites (20). Deux groupes de veaux ont reçu le seul vaccin pestique, deux autres ont reçu le vaccin mixte antipestique-antipéripneumonique (souche $\mathrm{KH}_{3} \mathrm{~J}$ ou souche $\mathrm{T}_{1}$ ).

Ces vaccins lyophilisés ont été reconstitués extemporanément soit en sérum physiologique, soit en solution molaire de sulfate de magnésium, sel apportant une thermo-stabilisation cationique au virus bovipestique (6). La dilution opérée a été celle préconisée pour l'utilisation de ces vaccins sur le terrain (généralement, reprise d'une pastille lyophilisée de $5 \mathrm{ml}$ dans $100 \mathrm{ml}$ de solvant).

Le tableau I explique le schéma d'expérience.

En complémentation d'essais antérieurement réalisés avec le virus morbilleux (21), un groupe de veaux a reçu ce virus, souche MB 113 Y adaptée à la culture en cellules bovines.

\section{Modes d'application}

Trois méthodes ont été essayées :

- l'aérosol vrai, produit avec un appareil médical générateur d'aérosol (*) et une colonne Halpern délivrant des particules de la taille du micron; un masque de polyéthylène dans lequel est engagé le mufle du bovin reçoit à son autre extrémité le tube de la colonne. Le générateur est mis en marche pendant 5 minutes. D'après le constructeur, la quantité de liquide brumisée pendant ce temps est de $1 \mathrm{ml}$;

- le dépôt de $5 \mathrm{ml} \mathrm{du} \mathrm{matériel} \mathrm{vaccinal}$ avec une sonde en matière plastique semirigide d'une dizaine de $\mathrm{cm}$ de long et d'un diamètre extérieur de $4 \mathrm{~mm}$, montée sur l'embout d'une seringue. La tête du veau est maintenue pendant l'opération en flexion supérieure de façon à ce que le liquide puisse se répandre dans les fosses nasales. Certains veaux ont par la suite des mouvements de déglutition et chez d'autres un peu de liquide reflue par les naseaux lorsqu'on les relâche;

- la vaporisation de la muqueuse nasale avec un vaporisateur de parfumerie en matière plastique souple, délivrant des gouttelettes; l'orifice de l'appareil est maintenu devant chacun des naseaux tandis que l'on presse le corps. Chaque veau reçoit de la sorte environ $1 \mathrm{ml}$ de vaccin brumisé dans chacune de ses cavités nasales. On espère ainsi éviter la déglutition du liquide en même temps que l'on couvre une plus grande surface de la muqueuse.

\section{Techniques sérologiques}

a) Séro-neutralisation bovipestique. On a suivi la méthodologie de PLOWRIGHT et FERRIS (15) de neutralisation en cultures cellulaires par la méthode "à virus constant sérum variable $"$.

$\left({ }^{\circ}\right)$ Jouan-Quetin, 163, avenue Gambetta, $75 \mathrm{~Pa}-$ ris $20 \mathrm{e}$. 
TABLEAU $\mathrm{N}^{\circ} \mathrm{I}$

Schéma d'expérience de vaccination antibovipestique par voie nasale

\begin{tabular}{|c|c|c|c|c|c|c|}
\hline Groupe & Animaux & Numéros & \multicolumn{2}{|c|}{ Vaccin et titre $\left(\mathrm{DCP}_{50} / \mathrm{m} 1\right)$} & $\begin{array}{c}\text { Mo d e } \\
d^{\text {tapplication }}\end{array}$ & Quantité \\
\hline 1 & Adultes & $\begin{array}{ll}2 & 561 \\
2 & 562 \\
3 & 920\end{array}$ & RPOK-BK (.) & $2,7(\ldots)$ & Aérosol & $1 \mathrm{~m} 1$ \\
\hline 2 & Veaux & $\begin{array}{rl}5 & 653 \\
& \text { à } \\
5 & 676 \\
\end{array}$ & $\begin{array}{l}\text { Mixte } \\
\text { RPOK-BK } \\
+\mathrm{KH}_{3} \mathrm{~J}\end{array}$ & 3,3 & $\begin{array}{l}\text { Sonde } \\
\text { plastique }\end{array}$ & $5 \mathrm{~m} 1$ \\
\hline 3 & Veaux & $\begin{array}{ll}5 & 129 \\
& \vdots \\
5 & 157\end{array}$ & RPOK-BK & 2,5 & Vaporisateur & $2 \mathrm{ml}$ \\
\hline 4 & Adultes & $\begin{array}{ll}5 & 001 \\
& \text { à } \\
5 & 010\end{array}$ & RPOK-BK & 2,5 & Vaporisateur & $2 \mathrm{ml}$ \\
\hline 5 & Veaux & $\begin{array}{ll}5 & 103 \\
& \mathrm{a} \\
5 & 127\end{array}$ & MB $113 \mathrm{Y}$ & 4,3 & $\begin{array}{l}\text { Sonde } \\
\text { plastique }\end{array}$ & $5 \mathrm{ml}$ \\
\hline 6 & Adultes & $\begin{array}{l}5006 \\
\text { à } \\
5 \text { D09 }\end{array}$ & MB $113 \mathrm{Y}$ & 4,3 & $\begin{array}{l}\text { Sonde } \\
\text { plastique }\end{array}$ & $5 \mathrm{~m} 1$ \\
\hline 7 & Veaux & $\begin{array}{ll}5 & 814 \\
& \grave{a} \\
5 & 846\end{array}$ & $\begin{array}{l}\text { Míxte RPOK-BK } \\
+\mathrm{T}_{1}\end{array}$ & 2,9 & $\begin{array}{l}\text { Sonde } \\
\text { plastique }\end{array}$ & $5 \mathrm{ml}$ \\
\hline B & Veaux & $\begin{array}{ll}5 & 551 \\
& \mathrm{a} \\
5 & 597\end{array}$ & $\begin{array}{c}\mathrm{RPOK}-\mathrm{BK} \\
\text { en } \\
\mathrm{SO}_{4} \mathrm{Mg}, 1 \mathrm{M}\end{array}$ & 2,9 & Vaporisateur & $2 \mathrm{~m} 1$ \\
\hline 9 & Veaux & $\begin{array}{ll}5 & 315 \\
& \text { à } \\
5 & 330\end{array}$ & $\begin{array}{l}\text { RPOK-BK } \\
\text { en } \\
\mathrm{SO}_{4} \mathrm{Mg}, 1 \mathrm{M}\end{array}$ & 2,9 & Vaporisateur & $2 \mathrm{~m} 1$ \\
\hline
\end{tabular}

(.) Souche de virus bovipestique attênué de Plowright et Ferris (14).

(..) Exprimé par 1 'exposant du $\log _{10}$ de la $\mathrm{DCP}_{50}$ de virus bovipestique ou morbilleux; cette quantité de virus représente ce qui est normalement contenu dans 1 dose vaccinale inoculêe par voie souscutanée.

b) Inhibition de l'hémagglutination morbilleuse. Elle est réalisée selon les normes de BÖGEL, ENDERS-RUCKLE et PROVOST (2) pour la recherche des anticorps antimorbilleux et antibovipestiques chez les bovins.

c) Immunofluorescence. La technique n'a été utilisée que pour la recherche qualitative des muco-anticorps nasaux; ses modalités (méthode indirecte, dite " du sandwich ») ont déjà été décrites (18).

\section{RESULTATS}

Les vaccins -délivrés par les procédés mentionnés jouissent d'autant d'innocuité que par voie sous-cutanée; tout au plus a-t-on pu remarquer une légère élévation (de $1^{\circ} \mathrm{C}$ ) pendant 48 heures de la température rectale des 3 adultes réceptifs du groupe 1 ayant reçu l'aérosol vaccinal.

Les résultats sérologiques seront maintenant examinés en détaíl. 
TABLEAU $\mathbb{N}^{0}$ II

Cinêtique des anticorps sêriques après un aérosol de vaccin bovipestique dêlivré à des zébus adu1tes (groupe 1)

\begin{tabular}{|c|c|c|c|c|}
\hline \multirow{3}{*}{$\begin{array}{c}\text { Numéro } \\
\text { des } \\
\text { zëbus }\end{array}$} & \multicolumn{4}{|c|}{ Anticorps antibovipestiques } \\
\hline & \multicolumn{2}{|c|}{ Avant aérosol } & \multicolumn{2}{|c|}{28 j après aérosol } \\
\hline & IHM & $\mathrm{SN}$ & IHM & $\mathrm{SN}$ \\
\hline 2561 & $<2$ & 0 & 32 & $>3$ \\
\hline 2562 & $<2$ & 0 & 16 & $>3$ \\
\hline 3920 & $<2$ & 0 & 4 & $>3$ \\
\hline
\end{tabular}

IHM : réaction d'inhibition de $1^{\prime}$ hémagglutination morbilleuse;

SN : réaction de séro-neutralisation bovipestique;

Les titres IHM sont exprimés par l'inverse de la fraction de la dilution du serum, les titres SN par

1 'exposant du $\log _{10}$ de cette dilution ( $\mathrm{TN}_{50}$ ). Il en sera de même dans tous les tableaux.

\section{Zébus adultes recevant le vaccin antipestique en aérosol (groupe 1)}

La séro-conversion est d'excellente qualité comme le montre le tableau II.

\section{Veaux recevant le vaccin antipestique par dépôt avec une sonde plastique (groupes 2 et 7 )}

Les tableaux III et IV rendent compte des résultats.
Dans le tableau III, on constate que sur 19 veaux contrôlés par séro-neutralisation après vaccination, 10 ont une séro-conversion (et vraisemblablement $12 \mathrm{si}$ l'on inclut les veaux 5658 et 5671 dont on peut penser, dans le contexte, qu'ils ne possédaient pas d'anticorps sériques ante-vaccinaux). Deux de ces montées d'anticorps sont de mauvaise qualité ( $\mathrm{N}^{\text {os }} 5663$ et 5668 ), ce qui reste intrigant pour ce dernier veau ne possédant pas d'immunité colostrale. Il n'y a qu'un seul échec vrai de la vaccination $\left(\mathrm{N}^{0} 5675\right)$.

TABLEAU $\mathrm{N}^{\circ}$ III

Cinétique des anticorps sériques après dépôt de vaccin bovipestique dans les cavités nasales à l'aide d'une sonde plastique (groupe 2)

\begin{tabular}{|c|c|c|c|c|c|}
\hline \multirow{3}{*}{ Numéros } & \multirow{3}{*}{$\begin{array}{c}\text { A g e } \\
\text { (en mois) }\end{array}$} & \multicolumn{4}{|c|}{ Anticorps sëriques } \\
\hline & & \multicolumn{2}{|c|}{ Avant vaccination } & \multicolumn{2}{|c|}{ Après vaccination } \\
\hline & & IHM & $\mathrm{SN}$ & IHM & $\mathrm{SN}$ \\
\hline $\begin{array}{r}5653 \\
54 \\
55 \\
56 \\
57 \\
58 \\
59 \\
61 \\
62 \\
63 \\
64 \\
65 \\
66 \\
67 \\
68 \\
69 \\
70 \\
71 \\
72 \\
73 \\
74 \\
75 \\
76\end{array}$ & $\begin{array}{rl}3 \\
2 \\
3 \\
4 \\
2 \\
6 \mathrm{tv}(.) \\
3 \\
2 \\
7 \mathrm{tv} \\
7 & \mathrm{tv} \\
2 & \\
7 & \mathrm{tv} \\
7 & \mathrm{tv} \\
3 & \\
3 & \\
4 \\
1 \\
9 \\
\mathrm{tv} \\
10 \mathrm{tv} \\
10 \mathrm{tv} \\
2 \\
2 \\
2\end{array}$ & $\begin{array}{l}<2 \\
<2 \\
<2 \\
<2 \\
<2 \\
<2 \\
<2 \\
<2 \\
<2 \\
<2 \\
<2 \\
<2 \\
<2 \\
<2 \\
<2 \\
<2 \\
<2 \\
<4 \\
<2 \\
<2 \\
<2 \\
<2 \\
<2\end{array}$ & $\begin{aligned}< & 0,3 \\
< & 0,3 \\
> & 1,2 \\
< & 0,3 \\
< & 0,3 \\
& \mathrm{NF}(. .) \\
> & 1,2 \\
< & 0,3 \\
< & 0,3 \\
& 0,9 \\
> & 1,2 \\
> & 1,2 \\
< & 0,3 \\
< & 0,3 \\
< & 0,3 \\
< & 0,3 \\
> & 1,2 \\
& 51, . . \\
> & 1,2 \\
> & 1,2 \\
> & 1,2 \\
< & 0,3 \\
< & 0,3\end{aligned}$ & $\begin{array}{l}<2 \\
<2 \\
<2 \\
<2 \\
<2 \\
<22 \\
<2 \\
<2 \\
<2 \\
<2 \\
<2 \\
<2 \\
<2 \\
<2 \\
<2 \\
<2 \\
<2 \\
\quad 4 \\
<2 \\
<2 \\
<2 \\
<2 \\
<2\end{array}$ & $\begin{aligned} & \mathrm{NF} \\
> & 1,2 \\
& \mathrm{NF} \\
& \mathrm{NF} \\
> & 1,2 \\
> & 1,2 \\
> & 1,2 \\
> & 1,2 \\
> & 1,2 \\
& 1,2 \\
> & 1,2 \\
> & 1,2 \\
> & 1,2 \\
> & 1,2 \\
& 0,6 \\
> & 1,2 \\
> & 1,2 \\
> & 1,2 \\
> & 1,2 \\
& \mathrm{NF} \\
& 0,9 \\
< & 0,3 \\
> & 1,2\end{aligned}$ \\
\hline
\end{tabular}

(.) trace auriculaire de vaccination, apposée à I'emporte-pièce;

(..) réaction non effectuẻe pour des raisons diverses;

(...) souillures bactériennes ou fungiques. 
TABLEAU N ${ }^{\circ}$ IV

Cinétique des anticorps antibovipestiques après dêpôt de vaccin antipestique dans les cavités nasales avec une sonde plastique (groupe 7)

\begin{tabular}{|c|c|c|c|c|}
\hline \multirow{2}{*}{ Numēros } & \multirow{2}{*}{$\begin{array}{c}\text { A g e } \\
\text { (en mois) }\end{array}$} & \multicolumn{2}{|c|}{ Anticorps gériques } & \multirow{2}{*}{$\begin{array}{l}\text { Muco-anticorps } \\
\text { (fluorescence) }\end{array}$} \\
\hline & & Avant vaccination & Après vaccination & \\
\hline 5814 & 5 & $<0,9$ & $\mathrm{NF}$ & - \\
\hline & 6 & $<0,9$ & 3,0 & - \\
\hline 16 & 6 & $<0,9$ & 2,7 & + \\
\hline 17 & $4-5$ & $<0,9$ & 3,0 & + \\
\hline 18 & 6 & $<0,9$ & 3,6 & $\operatorname{Tr}$ \\
\hline 19 & 2 & 1,2 & $\mathrm{NF}$ & - \\
\hline 20 & 6 & $<0,9$ & $\mathrm{NF}$ & \\
\hline 21 & 7 & $<0,9$ & 2,7 & + \\
\hline 22 & 3 & $<0,9$ & 2,7 & + \\
\hline 23 & 3 & $<0,9$ & 0,9 & + \\
\hline 24 & 4 & $<0,9$ & $>3,0$ & - \\
\hline 25 & 6 & $<0,9$ & $\mathrm{NE}$ & \\
\hline 26 & 5 & $>2,1$ & $>3,0$ & + \\
\hline 27 & 2 & 1,5 & $\mathrm{NF}$ & \\
\hline 28 & 2 & $>2,1$ & $\mathrm{NF}$ & \\
\hline 29 & 3 & 0,9 & 0,3 & \\
\hline 30 & 3 & $<0,9$ & 1,5 & \\
\hline 31 & 2 & 1,2 & 0,9 & \\
\hline 32 & 4 & $<0,9$ & 2,4 & - \\
\hline 33 & 1 & $<0,9$ & NF & \\
\hline $\begin{array}{l}34 \\
35\end{array}$ & $\begin{array}{l}5 \\
1\end{array}$ & $\begin{array}{r}<0,9 \\
1,2\end{array}$ & $\begin{array}{l}2,4 \\
1,2\end{array}$ & + \\
\hline 36 & 2 & $<0,9$ & 2,7 & \\
\hline 37 & 5 & 1,8 & $>3,0$ & \\
\hline 38 & 3 & $<0,9$ & $\mathrm{NF}$ & \\
\hline 39 & 4 & $<0,9$ & $\mathrm{NF}$ & \\
\hline $\begin{array}{l}40 \\
41\end{array}$ & $\begin{array}{l}3 \\
2\end{array}$ & $\begin{array}{r}<0,9 \\
0,9\end{array}$ & $\begin{array}{l}2,1 \\
2,1\end{array}$ & $\overline{+}$ \\
\hline 42 & 3 & $<0,9$ & 3,0 & - \\
\hline 43 & 5 & $<0,9$ & 3,6 & + \\
\hline 44 & 4 & $<0,9$ & 3,0 & - \\
\hline 45 & 2 & $>2,1$ & 2,7 & \\
\hline 46 & 5 & $<0,9$ & 3,0 & \\
\hline
\end{tabular}

Dans le groupe 7 (tableau IV), il existe, chez les 24 veaux contrôlés après vaccination, 19 séro-conversions (et même très vraisemblablement 20 en comptabilisant le veau 5826 chez qui sont apparus des muco-anticorps en dépit d'un important titre sérique ante-vaccinal). Il y a un échec de vaccination pour le 5829 , de même que pour le veau 5823 dont la séro-conversion peut être jugée de trop faible ampleur par rapport à celle de ses congénères.

Au total, pour les deux groupes, c'est une proportion de 72 p. 100 des veaux chez lesquels la vaccination par voie nasale a été efficace. A l'examen du tableau IV, il apparaît que c'est autour du titre $\mathbf{T N}_{50}=0,9$ en anticorps sériques ante-vaccinaux que se situent les chances de prise vaccinale; il n'existe alors que 3 veaux ( $\mathrm{N}^{\text {ов }} 5829,5831$ et 5835 ) pour lesquels il n'y a pas de montée d'anticorps alors que d'autres ( $N^{\text {os }} 5826,5837,5841$ et 5845), avec un important reliquat d'anticorps colostraux, accusent néanmoins une nette séroconversion. Le pourcentage de succès de la prise vaccinale atteint alors 86,9 p. 100 en fixant le seuil d'efficacité à $\mathrm{TN}_{50} \leqslant 0,9$; il paraît intéressant de relever que certains animaux ont une montée d'anticorps très nette malgré l'existence d'un titre important avant la vaccination nasale.

Des muco-anticorps nasaux sont apparus chez 10 veaux sur 18 desquels on a pu obtenir le mucus nasal; ce résultat sera commenté plus loin.

\section{Veaux et zébus adultes recevant le vaccin antipestique par vaporisation (groupes 3, 4, 8 et 9)}

La vaporisation de la muqueuse nasale des zébus adultes sensibles à la peste entraîne pour eux une séro-conversion pestique, preuve de la réplication du virus (tableau $\mathrm{V}$ ). 
TABLEAU N $\mathrm{N}^{\circ} \mathrm{V}$

Séro-conversion des zêbus adultes après vaporisation

de la muqueuse nasale avec le vaccin antibovipestique (groupe 4)

\begin{tabular}{|c|c|c|c|c|}
\hline \multirow{3}{*}{$\begin{array}{c}\text { Numéro } \\
\text { des } \\
\text { zébus }\end{array}$} & \multicolumn{4}{|c|}{ Anticorps antivovipestiques } \\
\hline & \multicolumn{2}{|c|}{ Avant vaporisation } & \multicolumn{2}{|c|}{ Après vaporisation } \\
\hline & IHM & $\mathrm{SN}$ & IHM & SN \\
\hline 5001 & $<2$ & 0 & $<2$ & + \\
\hline 5010 & $<2$ & 0 & $<2$ & + \\
\hline
\end{tabular}

Pour les veaux, les résultats diffèrent selon qu'ils hébergent ou non des anticorps colostraux (tableaux VI et VII).

Sur 29 veaux contrôlés du groupe 3, dix accusent une montée d'anticorps après la vaccination; toutefois, on ne possède que 24 sérums d'avant la vaporisation, ce qui donne une proportion de prise vaccinale de 41,6 p. 100. On notera que si un veau $\left(\mathrm{N}^{\circ} 5136\right)$ a répondu positivement malgré la présence d'anticorps colostraux de titre $\mathbf{T N}_{50}=1,2$ et un autre ( $\mathrm{n}^{\circ}$ 5137) avec un reliquat d'anticorps à $\mathrm{TN}_{\overline{5} 0}=0,6$ il existe un échec de vaccination pour le $\mathrm{n}^{\circ} 5145$ alors que les veaux 5132 et 5154 voient une montée de leurs anticorps antipestiques. Deux autres veaux (n ${ }^{\text {os }} 5130$ et 5143) n'ont pas eu de séro-conversion avec un reliquat d'anticorps à $\mathrm{TN}_{50}=0,9$.

Les résultats d'ensemble des groupes 8 et 9 sont meilleurs : on observe 47 séro-conversions ou montée d'anticorps pour 56 veaux dont on est sûr qu'ils n'ont pas été vaccinés ou qui n'ont que des reliquats d'anticorps. La prise vaccinale est de 85,6 p. 100. Il existe là

TABLEAU $\mathrm{N}^{\circ} \mathrm{VI}$

Cinêtique des anticorps antiboyipestiques chez des veaux après vaporisation de la muqueuse nasale avec le vaccin antibovipestique (groupe 3 )

\begin{tabular}{|c|c|c|c|c|c|}
\hline \multirow{2}{*}{$\begin{array}{c}\text { Numéro } \\
\text { des } \\
\text { veaux }\end{array}$} & \multirow{2}{*}{$\begin{array}{c}\text { A g e } \\
\text { (en mois) }\end{array}$} & \multicolumn{2}{|c|}{ Anticorps avant vaccination } & \multicolumn{2}{|c|}{ Anticorps après vaccination } \\
\hline & & IHM & $\mathrm{SN}$ & IHM & SN \\
\hline $\begin{array}{r}529 \\
30 \\
31 \\
32 \\
33 \\
34 \\
35 \\
36 \\
37 \\
38 \\
39 \\
40 \\
41 \\
42 \\
43 \\
44 \\
45 \\
46 \\
47 \\
48 \\
49 \\
50 \\
51 \\
52 \\
53 \\
54 \\
55 \\
56 \\
57\end{array}$ & $\begin{array}{r}12 \\
12 \\
8 \\
6 \\
8 \\
12 \\
8 \\
8 \\
12 \\
6 \\
4 \\
4 \\
4 \\
4 \\
3 \\
12 \\
4 \\
10 \\
2 \\
3 \\
6 \\
2 \\
10 \\
10 \\
12 \\
7 \\
1 \\
1 \\
2\end{array}$ & $\begin{array}{l}<2 \\
<2 \\
<2 \\
<2 \\
<2 \\
<2 \\
<2 \\
<2 \\
<2 \\
<2 \\
<2 \\
<2 \\
<2 \\
<2 \\
<2 \\
<2 \\
<2 \\
<2 \\
<2 \\
<2 \\
<2 \\
<2 \\
<2 \\
<2 \\
<2 \\
<2 \\
<2 \\
<2 \\
<2\end{array}$ & $\begin{aligned}> & 1,2 \\
& 0,9 \\
> & 1,2 \\
& 0,3 \\
> & 1,2 \\
< & 0,3 \\
> & 1,2 \\
& 1,2 \\
& 0,6 \\
& N F \\
> & 1,2 \\
> & 1,2 \\
< & 0,3 \\
& N F \\
& 0,9 \\
< & 0,3 \\
& 0,3 \\
& N F \\
> & 1,2 \\
> & 1,2 \\
< & 0,3 \\
& N F \\
& N F \\
< & 0,3 \\
< & 0,3 \\
& 0,3 \\
> & 1,2 \\
< & 0,3 \\
< & 0,3\end{aligned}$ & $\begin{array}{l}<2 \\
<2 \\
<2 \\
<2 \\
<2 \\
<2 \\
<2 \\
<2 \\
<2 \\
<2 \\
<2 \\
<2 \\
<2 \\
<2 \\
<2 \\
<2 \\
<2 \\
<2 \\
<2 \\
<2 \\
<2 \\
<2 \\
<2 \\
<2 \\
<2 \\
<2 \\
<2 \\
<2 \\
<2\end{array}$ & 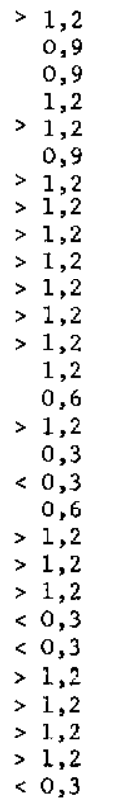 \\
\hline
\end{tabular}


TABLEAU $N^{\circ} \mathrm{VII}$

Cinétique des anticorps antibovipestiques chez des veaux recevant

le vaccin antipestigue par vaporisation de la muqueuse nasale (groupes 8 et 9)

\begin{tabular}{|c|c|c|c|c|}
\hline \multirow{2}{*}{ Numéros } & \multirow{2}{*}{$\begin{array}{c}\text { A g e } \\
\text { (en mois) }\end{array}$} & \multicolumn{2}{|c|}{ Anticorps sëriques } & \multirow{2}{*}{$\begin{array}{r}\text { Muco-Anticorps } \\
\text { (flvorescence) }\end{array}$} \\
\hline & & Avant vaccination & Après vaccination & \\
\hline 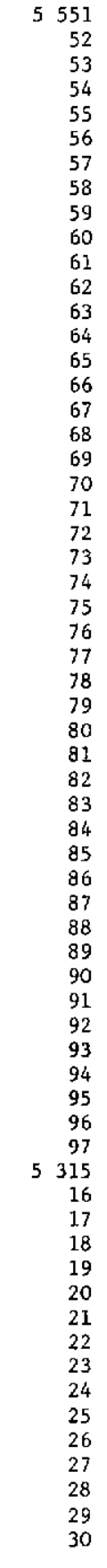 & 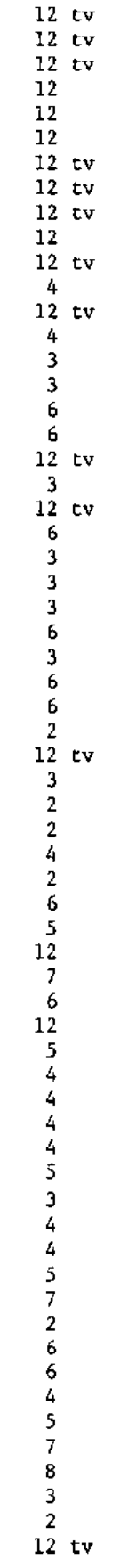 & 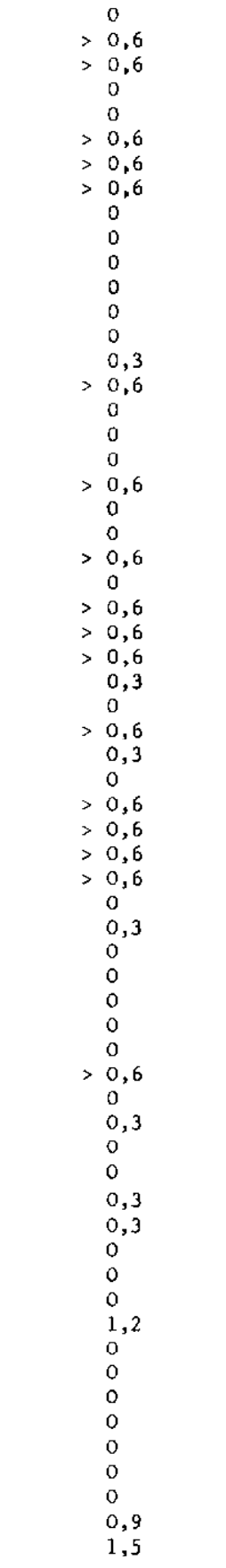 & 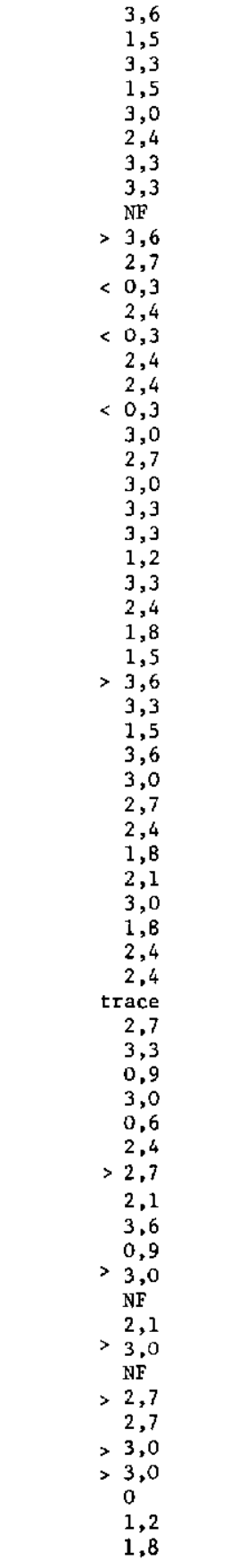 & $\begin{array}{l}+ \\
- \\
+ \\
+ \\
\mathrm{NF} \\
+ \\
+ \\
\mathrm{NF} \\
- \\
+ \\
+ \\
- \\
+ \\
\mathrm{NF} \\
-\end{array}$ \\
\hline
\end{tabular}


encore des échecs vrais de la vaccination $\left(\mathrm{n}^{\text {os }} 5562,5564,5567\right)$ ou une mauvaise séroconversion ( $\mathrm{n}^{\text {10s }} 5591$ et 5596). Cependant, comme pour le groupe 7 , de nombreux veaux hébergeant des anticorps colostraux ont des titres en anticorps sériques post-vaccinaux de très bonne qualité. Le seuil des anticorps colostraux assurant le succès de la prise vaccinale paraît, là encore, se situer à $\mathbf{T N}_{50} \leqslant 0,9$.
Les muco-anticorps du mucus nasal ne reflètent pas entièrement la prise vaccinale; ce point sera commenté.

\section{Veaux et zébus adultes}

recevant le virus morbilleux par dépôt avec une sonde plastique (groupes 5 et 6)

TABLEAU $\mathrm{N}^{\mathrm{a}} \mathrm{VIII}$

Séro-conversion des zébus adultes après dêpôt à la sonde plastique du virus morbilleux $\mathbb{M N} 113 \mathrm{Y}$ (groupe 6).

\begin{tabular}{|c|c|c|c|c|}
\hline \multirow{2}{*}{$\begin{array}{c}\text { Numéro } \\
\text { des } \\
\text { zébus }\end{array}$} & \multicolumn{4}{|c|}{ A n t i c or s s } \\
\cline { 2 - 5 } & \multicolumn{2}{|c|}{ Avant intervention } & \multicolumn{2}{|c|}{ Après intervention } \\
\hline & IHM & SN & IHM & SN \\
\hline 5006 & 2 & 0 & 2 & + \\
5009 & 2 & 0 & 2 & + \\
\hline
\end{tabular}

Le tableau VIII indique que les 2 zébus adultes sensibles à la peste ont une séro-conversion.

Pour les veaux, il est apparent que la vaccination hétérologue par voie nasale entraîne la séro-conversion antipestique de ceux ne possédant pas d'anticorps colostraux (comme le 5113) mais également de ceux qui en hébergent à un $\mathrm{TN}_{50} \leqslant 0,9$ (veaux 5107,5109 , 5110,5111 et 5123 ); le veau 5105 , situé à la limite, n'a pas répondu à la vaccination. On ne peut rien conclure pour les autres étant donné la conduite de l'expérience.

\section{Epreuves bovipestiques virulentes}

Les 7 zébus adultes ayant reçu, soit l'aérosol, soit le dépôt de vaccin antipestique, soit celui de virus morbilleux sont éprouvés par un aérosol de virus bovipestique délivré dans des conditions déjà décrites (22). Tous résistent.

Quatre veaux $(5554,5556,5558$ et 5826$)$, achetés avec grandes difficultés à leurs propriétaires, ont été éprouvés par voie souscutanée avec une suspension de rate pestique. Tous quatre ont résisté. Toutefois, étant donné l'anamnèse, il paraît difficile de conclure pour le veau 5558 qui avait une trace auriculaire de vaccination avant l'intervention.

\section{Remarque sur les anticorps inhibant l'hémagglutination morbilleuse}

Si l'on excepte les bovins du groupe 1 pour lesquels l'essai a été réalisé deux ans avant les autres, il est apparent que pour aucun autre animal la vaccination pernasale n'a été suivie de montée d'anticorps inhibant l'hémagglutination morbilleuse; la simple trace du $\mathrm{n}^{\circ} 5110$ (tableau IX) n'est pas convaincante. Bien plus, sur l'ensemble des veaux possédant des anticorps colostraux, deux seulement $\left(\mathrm{n}^{\text {ns }} 5671\right.$ et 5103) possèdent une activité sérique inhibant l'hémagglutination morbilleuse. C'est une proportion bien plus faible que celle qui avait été relevée dans une précédente expérience (21), dans laquelle 6 p. 100 des veaux possédaient des anticorps IHM.

L'absence d'immunogénèse morbilleuse après vaccination nasale contraste donc avec celle de résultats antérieurement acquis $(13,23)$ dans lesquels 72 et 82 p. 100 respectivement des bovins vaccinés par voie sous-cutanée avec Ie même virus de culture cellulaire présentaient une séro-conversion morbilleuse un mois après l'intervention.

\section{DISCUSSION}

L'indépendance des immunités post-infectieuses ou post-vaccinales humorales et nasales 
TABLEAU $N^{\circ} I X$

Cinétique des anticorps chez des veaux recevant le virus morbilleux MB 113 Y par dépôt dans les cavités nasales avec une sonde plastique (groupe 5).

\begin{tabular}{|c|c|c|c|c|c|}
\hline \multirow{3}{*}{ Numéros } & \multirow{3}{*}{$\begin{array}{c}A g \mathrm{e} \\
\text { (en mois) }\end{array}$} & \multicolumn{4}{|c|}{ Anticorps sériques } \\
\hline & & \multicolumn{2}{|c|}{ Avant vaccination } & \multicolumn{2}{|c|}{ Après vaccination } \\
\hline & & IHM & SN & IHM & SN \\
\hline $\begin{array}{r}5103 \\
04 \\
05 \\
06 \\
06 \\
07 \\
08 \\
09 \\
10 \\
11 \\
12 \\
13 \\
14 \\
15 \\
16 \\
17 \\
18 \\
19 \\
20 \\
21 \\
22 \\
23 \\
24 \\
25 \\
26 \\
27\end{array}$ & $\begin{array}{c}2 \\
1 \\
2 \\
2 \\
1,5 \\
2 \\
3 \\
4 \\
4 \\
12 \\
5 \\
2 \\
9 \text { jours } \\
25 \text { jours } \\
9 \text { jours } \\
2 \\
3 \\
2 \\
1 \\
1 \\
6 \\
1 \\
2 \text { jours } \\
4 \\
2\end{array}$ & $\begin{aligned} & 2 \\
< & 2 \\
< & 2 \\
< & 2 \\
< & 2 \\
< & 2 \\
< & 2 \\
< & 2 \\
< & 2 \\
< & 2 \\
< & 2 \\
< & 2 \\
< & 2 \\
< & 2 \\
< & 2 \\
< & 2 \\
< & 2 \\
< & 2 \\
< & 2 \\
< & 2 \\
< & 2 \\
< & 2 \\
< & 2 \\
< & 2 \\
< & 2\end{aligned}$ & $\begin{aligned}> & 1,2 \\
> & 1,2 \\
& 0,9 \\
> & 1,2 \\
& 0,9 \\
> & 1,2 \\
& 0,6 \\
- & 0,6 \\
& 0,6 \\
> & 1,2 \\
& 0 \\
> & 1,2 \\
> & 1,2 \\
> & 1,2 \\
> & 1,2 \\
> & 1,2 \\
& 0,6 \\
& 1,2 \\
> & 1,2 \\
> & 1,1 \\
& 0,6 \\
> & 1,2 \\
> & 1,2 \\
> & 1,2 \\
> & 1,2\end{aligned}$ & $\begin{array}{l}<2 \\
<2 \\
<2 \\
<2 \\
<2 \\
<2 \\
<2 \\
<2 \\
\text { trace } \\
<2 \\
<2 \\
<2 \\
<2 \\
<2 \\
<2 \\
<2 \\
<2 \\
<2 \\
<2 \\
<2 \\
<2 \\
<2 \\
<2 \\
<2 \\
<2 \\
<2 \\
<2\end{array}$ & $\begin{aligned}> & 1,2 \\
> & 1,2 \\
& 0,9 \\
> & 1,2 \\
> & 1,2 \\
& 1,2 \\
> & 1,2 \\
> & 1,2 \\
> & 1,2 \\
> & 1,2 \\
& 0,9 \\
> & 1,2 \\
> & 1,2 \\
> & 1,2 \\
> & 1,2 \\
> & 1,2 \\
& \mathrm{~N} F \\
> & 1,2 \\
> & 1,2 \\
> & 1,2 \\
> & 1,2 \\
> & 1,2 \\
> & 1,2 \\
> & 1,2 \\
> & 1,2\end{aligned}$ \\
\hline
\end{tabular}

est un fait maintenant bien établi chez différentes espèces animales. Pour ne citer que des travaux récents, ceux de DUNCAN et THOMSON $(7,8)$ pour l'infection à Pasteurella hemolytica et ceux de GUTEKUNST, PATON et VOLONEC (11) pour l'infection à virus parainfluenza 3 l'ont clairement démontré chez les bovins. Par une autre voie d'abord du problème, c'est également à cette notion que nous étions arrivés; c'est elle qui a déterminé la présente série d'expériences $(18,23)$.

La première question à résoudre était celle de la réceptivité des voies aériennes supérieures au virus bovipestique atténué de cultures cellulaires. En effet, une expérience déjà ancienne nous avait montré que certains des virus atténués par passages hétérologues infectaient de façon inconstante lorsqu'ils étaient donnés par aérosol (17). De plus, on pouvait émettre des doutes pour le virus de cultures cellulaires en se basant sur l'opinion de TAYLOR et PLOWRIGHT (25), selon Iaquelle ce virus-vaccin avait perdu tout caractère épithéliotrope et ne se répliquait que dans les cellules lymphatiques. Sans aucune- ment préjuger des sites de réplication du virus qui restent à démontrer, les résultats des présentes expériences indiquent clairement que l'infection nasale entraîne une séro-conversion humorale de titre élevé; pragmatiquement, c'est ce que l'on voulait obtenir. Ce résultat confirme un essai préliminaire de SINGH et collab. (24) qui avaient réussi à immuniser deux bovins par dépôt de virus-vaccin sur la muqueuse nasale.

Lorsque l'on replace les résultats de nos essais auprès de ceux obtenus en immunologie comparée, ils valent d'être commentés. L'efficacité de la vaccination pernasale est un fait bien établi dans la maladie de Newcastle. Elle est entrée dans la pratique courante, soit par dépôt de vaccin dans les narines (10), soit par aérosolisation (" mist-vaccine »). Elle est utilisée pour la vaccination anticarré d'espèces difficilement maniables comme le furet ou le renard (3). Les expériences menées avec le virus de la rougeole chez l'enfant sont, par contre, loin d'être démonstratives $(1,28)$ et malgré le succès de certains cliniciens $(5,26)$, la vaccination antimorbilleuse pernasale n'est pas entrée dans la pratique. 
On peut se demander si, plus que la voie d'introduction, ce ne sont pas des caractéristiques propres à la souche utilisée qui interviennent. On notera dans cette optique que nos essais ont été réalisés avec un virus bovipestique de bas passage adapté aux cellules bovines. Ce point mérite une investigation plus poussée.

Il n'en reste pas moins que si pratiquement l'administration pernasale de l'un ou l'autre des virus-vaccins est efficace, cette voie d'introduction est une grosse consommatrice de virus puisque, pour tenter de couvrir toute la surface de la muqueuse, on est conduit à brumiser ou à inoculer de 2 à 5 fois plus de vaccin que par voie sous-cutanée. Au regard des avantages concédés qui seront commentés plus loin, il apparaît que le reproche est mineur et que le prix du vaccin n'entre que peu en ligne de compte lorsque l'on peut briser la barrière humorale de l'immunité colostrale résiduelle des yeaux.

Ce dernier point est digne d'intérêt. L'impuissance dans laquelle sont plongés les planificateurs des campagnes de vaccination en face de ce problème a conduit à la recherche de l'utilisation possible d'un vaccin hétérologue, en l'occurence le virus morbilleux MB 113 Y (21). Sur le terrain, pourtant, on se contente de vacciner les veaux quel que soit leur âge, se réservant d'apposer la marque de vaccination lors d'une seconde intervention réalisée en un temps ultérieur, lorsqu'ils auront à coup sûr perdu leurs anticorps colostraux.

On pouvait se demander si la voie d'administration pernasale n'apporterait pas de solution, au moins partielle, à ce problème de l'immunisation des veaux.

Pour la compréhension des résultats, les données des tableaux III, IV, VI et VII ont été regroupées dans le tableau X. On n'y a

TABLEAU $\mathrm{N}^{\circ} \mathrm{X}$

\begin{tabular}{|c|c|c|}
\hline $\begin{array}{c}\text { Montée } \\
\text { d'anticorps }\end{array}$ & $\begin{array}{c}\text { Titre sérique } \\
\text { résidue1 }\end{array}$ & $\begin{array}{c}\text { Pas de montée } \\
\text { d'anticorps }\end{array}$ \\
\hline 41 & $<0,3$ & 8 \\
10 & 0,3 & 0 \\
1 & 0,6 & 0 \\
20 & $<0,9$ & 3 \\
2 & 3,2 & 2 \\
3 & $>1,2$ & 3 \\
\hline
\end{tabular}

inclus que les animaux pour lesquels la teneur en anticorps était connue de façon certaine avant et après l'intervention.

Il est apparent qu'il existe 8 échecs authentiques de la vaccination, ceux des veaux sans anticorps qui n'ont élaboré aucune immunité. On ne peut dire pour eux s'il s'agit d'un manque de réceptivité ou d'une faute dans le manuel opératoire; $a$ priori, et étant donné l'ensemble des résultats, il paraît que cette dernière hypothèse est la bonne. Si l'on exclut ces animaux du calcul, on s'aperçoit qu'au titre sérique seuil de $\mathrm{TN}_{50} \leqslant 0,9,72$ veaux sur 75 , soit 96 p. 100 , accusent une séroconversion après vaccination nasale (chiffre qui, au demeurant, ne tombe qu'à 86 p. 100 si l'on comptabilise les 8 échecs). A $\mathbf{T N}_{50}>0,9$ l'immunisation est plus aléatoire puisqu'elle tombe à 50 p. 100 ; il est tout de même remarquable que certains veaux possédant une forte immunité sérique colostrale répondent par une montée d'anticorps. Pareille chose ne s'observe jamais lors des vaccinations parentérales, où de simples traces d'anticorps colostraux résiduels entravent l'immunogénèse $(16,24)$. Au total, l'immunisation par voie pernasale permet l'immunisation active de nombre de veaux encore sous le couvert de leur immunité colostrale. II resterait à savoir quelles sont la durée et la qualité de cette immunité car PLOWRIGHT et TAYLOR (16) ont montré que des bovins possédant des traces d'anticorps sériques colostraux voyaient décliner rapidement leur immunité active. Tel pourrait être le cas de certains de nos veaux d'expérience $\left(\mathrm{n}^{\mathrm{os}} 5668,5830,5134,5552,5554,5573,5577\right.$, $5580,5594,5596$ et 5329); beaucoup d'autres, par contre, atteignent des titres d'excellente qualité.

Il paraît dès lors recommandable de ne pas hésiter à utiliser le procédé de vaccination pernasale pour vacciner les veaux plutôt que d'utiliser la voie sous-cutanée conventionnelle. Les quelques veaux qui seront "ratés" pour des raisons diverses seront retrouvés l'année d'après lors du passage annuel des équipes vaccinales. Ce passage annuel régulier garantit d'ailleurs le rattrapage des animaux qui perdraient leur immunité active en même temps que, par l'emploi d'un vaccin mixte (20), il assure la protection antipéripneumonique.

Il paraît difficile de recommander l'utilisation d'une sonde plastique plutôt que du vaporisa- 
teur; les résultats acquis avec run et l'autre sont égaux. Tout au plus, peut-on dire que le vaporisateur consomme moins de virus.

Le procédé de vaccination pernasale a été utilisé dans d'autres circonstances pour l'immunisation des jeunes encore sous le couvert de l'immunité maternelle. OTT et GORHAM (10) ont réussi à vacciner ainsi de jeunes furets âgés de 36 à 47 jours, mais récemment, FARRELL et collab. (9) n'ont pas vu de supériorité à l'aérosol par rapport à la voie sous-cutanée; il est possible que les particules de l'aérosol, pénétrant plus avant dans l'arbre respiratoire, soient neutralisées par la lymphe pulmonaire. Les poussins issus de poules vaccinées contre la maladie de Newcastle sont protégés Iorsque le vaccin B 1 est appliqué par voie nasale (27).

Il reste un point dogmatique important à élucider pour expliquer le succès de la vaccination antibovipestique des veaux par voie pernasale : c'est celui des sites de réplication du virus. Il est difficile de penser aux amygdales qui sont baignées de lymphe plasmatique chariant des anticorps. Doit-on songer alors aux petits follicules lymphoïdes de la muqueuse nasale ou tout simplement à son épithélium qui, lui, n'est pas au contact de muco-anticorps pestiques? Seule une recherche à venir peut éclaircir ce point.

De cet essai, il reste troublant que l'apparition de muco-anticorps pestiques n'a pas été détectée de façon aussi constante que celle à laquelle on pouvait s'attendre (tableaux IV et VII) chez des veaux présentant pourtant une conversion sérique post-vaccinale. Une explication logique, elle aussi à vérifier, vient à l'esprit. Les immuno-globulines des sécrétions nasales bovines sont principalement des Ig A (12). Or, dans la technique indirecte de révélation des muco-anticorps utilisée, on s'est servi d'une globuline fluorescente de lapin antiglobuline sérique de bœuf. Les immunoglobulines sériques du bœuf sont à prédominance d'Ig $G$ et d'Ig M; c'est donc dire que la globuline fluorescente de lapin employée ne devait contenir que très peu d'anticorps anti-Ig $\mathrm{A}$, globulines qui sont précisément celles que l'on cherchait à mettre en évidence. Il paraît intéressant de produire une globuline fluorescente anti-mucus nasal pour une telle recherche. On remarquera que la critique qui vient d'être faite n'entache pas d'erreur les résultats antérieurement acquis sur l'absence d'immunoglobulines de transfert dans le mucus nasal des veaux sous immunité colostrale (18). Les globulines du colostrum sont des Ig G 1 (4) qui auraient été détectés par la globuline fluorescente utilisée.

Le dernier point à commenter est celui de l'utilisation du virus morbilleux MB 113 Y par voie pernasale.

Il paraît, dans cet essai, ne pas donner de résultats supérieurs au virus-vaccin antipestique. En conséquence, son emploi n'a pas à être recommandé. On a retrouvé le comportement immunologique particulier déjà signalé (21) de cette souche qui, authentique virus de la rougeole à l'origine, détermine la génèse d'anticorps antipestiques vrais à défaut d'anticorps antimorbilleux.

\title{
SUMMARY
}

Trials of rinderpest nasal vaccination of calves with or without colostral immunity

\begin{abstract}
Based on the established knowledge that colostral antibodies of calves with maternal-derived immunity are not found in the nasal mucus, the authors have made nasal vaccination trials of such calves using either the tissue culture rinderpest vaccine or the MB 113 Y measles virus applied with a plastic tubing or a perfume hand-sprayer. This vaccination procedure allows the active immunisation of $96 \mathrm{p}$. 100 of calves with a residual antibody tıter $\mathbf{T N}_{\text {io }} \leqslant 0,9$ but also of some of those with higher titer. The nasal vaccination procedure has hence to be put into practice to break down the epizootiological cycle of rinderpest by reducing the number of disease susceptible calves and giving a local immunity which restrains viral replication.
\end{abstract}




\section{RESUMEN}

\section{Ensayos de vacunación contra la peste bovina por via pemasal de terneros con o sin immunidad calostral}

A partir de la observación establecida según la cual no se encuentran los anticuerpos del calostro en los muco-anticuerpos nasales de los terneros que siguen teniendo la inmunidad maternal transmitida, los autores vacunaron dichos animales por via nasal con el virus-yacuna antipestico de cultivos celulares o el virus morbilloso cepa MB $113 \mathrm{Y}$ aplicado mediante una sonda de plástico o un vaporizador. $\mathrm{El}$ procedimiento permite la inmunización activa de 96 p. 100 de los terneros aun teniendo un título serico residual de $\mathrm{TN}_{\text {so }} \leqslant 0,9$ y de algunos animales con más anticuerpos. Es útil que sea vulgarizado el método para interrumpir el ciclo epizootiologico de la peste bovina con reducir el número de terneros receptivos a la enfermedad al producir una inmunidad local que prohibe la replicación del virus.

\section{BIBLIOGRAPHIE}

1. BLACK (F. L.) et SHERIDAN (S.R.), Studies on an attenuated measles-virus vaccine. IV. Administration of vaccine by several routes, New Engl. J. Med., 1960, 263: 165-69.

2. BÖGEL (K.), ENDERS-RUCKLE (G.) et PROVOST (A.), Une réaction sérologique rapide de mesure des anticorps antibovipestiques, C.R. Acad. Sci., Paris, 1964, 259: 482 .

3. BROZEIT (H. E.) et ACKERMANN (O.), Die Anwendung von Staupe-Aerosol-Vakzine zur aktiven Immunisierung von Farmfüchsen, Kleintierpraxis, 1969, $7: 200-202$.

4. BUTLER (J.E.), KIDDY (C. A.), MAXWELL (C. F.), HYLTON (M. B.) et ASOFSKY (R.), Synthesis of immunoglobulins by yarious tissues of the cow, J. Dairy Sci., 1971, 54:1323-24.

5. CHIN-YUN LEE (G.), Intranasal vaccination with attenuated measles virus, Proc. Soc. Exp. Biol. Med., 1963, 112 : 656-58.

6. Connaissances acquises récemment sur la peste bovine et son virus, Rev. Elev. Méd. vét. Pays trop., 1966, 19 : 365-413.

7. DUNCAN (J. R.) et THOMSON (R.G.), Preliminary observations on the effect of specific immunity on nasal bacterial flora, Canad. J. comp. Med., 1970, 34 : 90-93.

8. DUNCAN (J. R.) et THOMSON (R. G.), Influence of immunization procedures on upper respiratory tract immunity in cattle, Canad. $J$. comp. Med., 1970, 34: 94-100.

9. FARRELL (R. K.), SKINWER (S. F.), GORHAM (J. R.) et LAVERMAN (L. H.), The aerosol and subcutaneous administration of attenuated egg adapted distemper vaccine to ferret kits from distemper immune females, Res. Vet. Sci., 1971, 12: $392-93$.

10. GORET (P.) et LE GALL (A), Méthodes et techniques des vaccinations locales, Encycl. vét. Pér., 1960, 15-16: 1-15.

11. GUTEKUNST (D. E.), PATON (I. M.) et VOLENEC (F. J.), Parainfluenza 3 vaccine in cattle : comparative efficacy of intranasal and intramuscular route, J. am. vet. med. Ass., 1969, 155: 1879-85.

12. MACH (J.P.), The bovine secretory immune system, J. dairy Sci., 1971, 54: 1327.

13. MAURICE (Y.), PROVOST (A.) et BORREDON (C.), Possibilités et limites de la réaction d'inhibition de l'hémagglutination morbilleuse dans la sérologie de la peste bovine, Rev. Elev. Méd. vét. Pays trop., 1969, 22 : 1-8.
14. PLOWRIGHT (W.) et FERRIS (R, D.), Studies with rinderpest virus in tissue culture. I. Growth and cytopathogenicity, J. comp. Path., 1959, 69 : 152-72.

15. PLOWRIGHT (W.) et FERRIS (R. D.), Studies with rinderpest virus in tissue culture. III. The stability of culture virus and its use in virus neutralization tests, Arch. Ges. Virusf., 1961, 11 : 516-33.

16. PLOWRIGHT (W.) et TAYLOR (W. P.), Long term studies of the immunity in East-african cattle following inoculation with rinderpest culture vaccine, Res. vet. Sci., 1967, 8: 118-28.

17. PROVOST (A.), Essai de transmission de la peste bovine par aérosol virulent, Bull. epizoot. Dis. Afr., 1958, 6 : 79-85.

18. PROVOST (A.), Observations sur les mucoanticorps nasaux des bovins, Rev. Elev. Méd. vét. Pays trop., 1970, 23 : 283-93.

19. PROVOST (A.), Transmission de la peste bovine par les veaux possédant une immunité antibovipestique colostrale, Rev. Elev. Méd. vét. Pays trop. (à paraître).

20. PROVOST (A.), BORREDON (C.) et QUEVAL (R.), Recherches immunologiques sur la péripneumonie. XII, Un vaccin mixte antibovipestique-antipéripneumonique inoculable en un seul temps, Bull. Off. Int. Epiz, 1969, 72 (1 a) : 165-203.

21. PROVOST (A.), MAURICE (Y.) et BORREDON (C.), Protection antibovipestique conférée aux bovins par le virus de la rougeole, Rev. Elev. Méd. vét. Pays trop., 1969, 21 : 145-64.

22. PROVOST (A.), MAURICE (Y.) et BORREDON (C.), Note sur la peste bovine expérimentale du dromadaire, Rev. Elev. Méd. vét. Pays trop., 1969, 21 : 293-96.

23. PROVOST (A.), MAURICE (Y.) et BORREDON (C.), Comportement clinique et immunologique lors de contamination bovipestique de bovins vaccinés depuis plusieurs années contre la peste bovine avec des vaccins de culture cellulaire, Rev. Elev. Méd. vét. Pays trop., 1969, 21 : 453-64.

24. SINGH (K.V.), OSMAN (O. A.), BAZ (T. A.) et ELCICY (I. F.), The use of tissue culture rinderpest vaccine for egyptian cattle and water buffaloes, Cornell vet., 1967, $57:$ 465-79.

25. TAYLOR (W. P.) et PLOWRIGHT (W.), Studies on the pathogenesis of rinderpest in experimental cattle. III. Proliferation of an attenuated strain in various tissues following subcutaneous inoculation, J. Hyg., Camb., 1965, 63 : 263-75. 
26. VEDA (S.), Studies on the combined use of killed and live measles vaccine. On the advantage of the inhalation method of live vaccine, Virus (Japan), 1966, 16: 16-28.

27. WHITE (H. F.), HANSON (E. L.) et ALBERTS (O. J.), Studies on Newcastle disease. X. Vacci- nation studies with Newcastle disease vaccine (intranasal type) in parentally immune and susceptible chicks, Poultry Sci., 1953, 32: 103-06.

28. ZDANOV (V.M.) et FADEEVA (L. L.), Problems of development of measles virus, Vop. Virus. (Moscou), 1959, 5: 51-57. 